ISSN 1997-5902

\title{
Analyse des caractéristiques structurelles et des performances technico-économiques de la riziculture irriguée en Côte d'Ivoire
}

\author{
${ }^{*}$ DOUMBIA Sékou et 2 DEPIEU Méougbé Ernest \\ 1 et 2CNRA, Km 17, route de Dabou ; Abidjan, Côte d'Ivoire ; Emails : moulouck2001@yahoo.fr; +225 07623053 ; \\ depieu@yahoo.fr ; +225 44831260
}

“Corresponding Author email: moulouck2001@yahoo.fr; Mobile: +225 07623053

Original submitted in on $17^{\text {th }}$ October 2013 Published online at www.m.elewa.org on $28^{\text {th }}$ February 2014. http://dx.doi.org/10.4314/jab.v74i1.3

\begin{abstract}
RESUME
Objectif : L'étude vise à analyser les caractéristiques structurelles et les performances technico-économiques de la riziculture irriguée en Côte d'Ivoire.

Méthodologie et résultats : Un diagnostic socio-économique a été conduit au cours du premier cycle de production de paddy en 2009 et a concerné un échantillon aléatoire de 200 riziculteurs répartis sur quatre départements au niveau des deux principales zones agro écologies du pays. Différents niveaux d'analyse ont été considérés, constitués d'une part des zones agro-écologiques et d'autre part des catégories d'origine sociale des producteurs. Les paramètres analysés sont la dotation en ressources productives, l'intensité de l'utilisation qui en est faite et les indicateurs de performance économique. Nos résultats indiquent des niveaux relativement faibles de performance ainsi que l'existence d'importantes disparités entre niveaux d'analyse due à des niveaux variables de dotation en ressources productives et à la diversité des stratégies de production. Conclusion et application des résultats : Cette étude révèle qu'une amélioration des performances d'ensemble en riziculture irriguée en Côte d'Ivoire passe par la prise en compte d'un certains nombre de dispositions comme (1) l'amélioration du niveau de formation des producteurs et la sensibilisation aux bonnes pratiques agricoles, et (2) l'amélioration de l'accès des riziculteurs aux intrants agricoles.

Mots clés : Diagnostic, riz irrigué, productivité, performances technico-économiques
\end{abstract}

Analysis of the structural characteristics, technical and economic performance of irrigated rice in Côte d'Ivoire

ABSTRACT

Objective: The study aims to analyze irrigated rice farms characteristics, and their technical and economic performances in Côte d'Ivoire.

Methodology and results: A socio economic survey was carried out, during the 2009 first cropping cycle, with 200 farmers randomly selected in four regions over the two major agro ecological zones (forest and savanna) of Côte d'Ivoire. Different levels of analysis were considered including the agro ecological zones and farmers' socioeconomic origins. Data was collected and analysis on economic characteristics such as farmers productive resources endowment and the intensity of use of those resources, productivity indicators, and global economic performances. This study results show relative poor economic performances of irrigated rice farming together with a large diversity of production strategies and level of input use. 
Conclusion and application of findings: This study reveals that improved overall performance in irrigated rice in Côte d'Ivoire through the inclusion of a number of provisions such as (1) improving the level of training producers and awareness of good agricultural practices, and (2) improving access to agricultural inputs rice. Key words: Diagnostic, irrigated rice, productivity, economic and technical performances

NB: 1 USD $=500$ FCFA

\section{INTRODUCTION}

En Côte d'Ivoire le riz est une culture vivrière de première importance. La production nationale est cependant loin de couvrir les besoins nationaux, et l'État recourt chaque année à des importations coûteuses en devises. En effet en 2009, les importations de riz se sont élevées à 919000 tonnes pour un coût estimé à plus de 235 milliards de FCFA (USD 470 000,000) (Anonyme, 2012). L'amélioration du niveau de production domestique apparaît comme une des solutions pour remédier à cette situation. Pour ce faire, les autorités ivoiriennes ont ciblé la riziculture irriguée comme le type de riziculture à promouvoir en priorité. Ce choix repose pour une bonne part sur le potentiel de production relativement élevé en écologie irriguée grâce à la plus ou moins bonne maîtrise de l'eau. En effet, le système rizicole ivoirien repose sur trois écologies d'inégales importance. II s'agit par ordre décroissant de la riziculture pluviale avec environ $85 \%$ des superficies, de la riziculture de bas fonds inondé, occupant environ $10 \%$ des superficies et de la riziculture irriguée qui compte seulement près de $5 \%$ des superficies. Produit en moyenne sur deux cycles, la riziculture irriguée contribue à hauteur de $20 \%$ environ à la production nationale de paddy estimée à 650000 tonnes, grâce à sa relative plus grande ouverture aux intrants modernes de production conjuguée à un niveau appréciable de

\section{METHODOLOGIE}

L'étude s'est déroulée au cours du premier cycle de culture du riz irrigué en 2009 dans les quatre départements de Gagnoa et de Daloa en zone forestière ; de Bouaké et de Korhogo en zone de savane connus pour l'importance de leur niveau de production de paddy. Une base de sondage a été constituée auprès des coopératives et nous en avons tiré un échantillon aléatoire de 200 producteurs, à raison de 50 riziculteurs par département.

Méthodes d'analyse : Le budget de culture a été établi en nous basant sur les prix des intrants et des produits en mécanisation. (Anonyme, 2012). Toutefois, les résultats de travaux antérieurs invitent à observer la plus grande prudence vis-à-vis de la priorité donnée à la riziculture irriguée, étant donné les expériences passées de production de riz en écologie irriguée en Côte d'Ivoire et dans de nombreux pays de la sousrégion. En effet, malgré les investissements importants dans les systèmes irrigués, la recherche et la vulgarisation, les coûts de la production rizicole irriguée en Afrique de l'ouest surpassent de manière générale les bénéfices. II découle de ce constat la nécessité de bien appréhender les conditions actuelles de production du paddy (Pearson et, 1981 ; Adesina, 1993). Notre diagnostic de la riziculture irriguée en Côte d'Ivoire, se veut une contribution à cet effort de compréhension. L'objectif général de l'étude est d'analyser le processus de production du paddy en écologie irriguée et de contribuer ainsi d'une part à la caractérisation des acteurs intervenant au niveau de la production et procéder d'autre part à l'estimation des paramètres technicoéconomiques. II s'agira dans une seconde phase, d'établir une situation de référence par rapport à quelques indicateurs clé et de formuler quelques recommandations par rapport à la politique d'appui à une spéculation cruciale en matière de sécurité alimentaire pour l'ensemble du pays.

vigueur au cours du premier cycle de culture du riz en 2009 ; les prix et les quantités indiquées correspondent à des estimations moyennes. Ce travail a été complété par l'analyse descriptive des structures d'exploitation. Le paddy a été acheté au producteur à $137 \mathrm{FCFA} / \mathrm{kg}$ (USD $0.274 / \mathrm{Kg}$ ), l'engrais a été vendu à $400 \mathrm{FCFA} / \mathrm{kg}$ (USD $0.8 / \mathrm{Kg}$ ), et 380 FCFA $/ \mathrm{kg}$ (USD 0.76/Kg), respectivement pour le NPK et l'urée aux producteurs. La semence de variété améliorée de riz irrigué, achetée à $600 \mathrm{FCFA} / \mathrm{kg}$ (USD 1.2/Kg), par les riziculteurs, est utilisée en moyenne pendant trois campagnes avant 

technico-économiques de la riziculture irriguée en Côte d'Ivoire

d'être renouvelée. II a été affecté un prix d'achat annuel de $200 \mathrm{FCFA} / \mathrm{kg}$ (USD 0.4/Kg), à cette semence. Le coût journalier de la main-d'œuvre salariée a été estimé à 1000 FCFA, (USD 2) la main-d'œuvre familiale a été valorisée au coût d'opportunité du travail. L'analyse a été menée au niveau agrégé, puis par zone agro écologique

\section{RESULTATS}

Caractéristiques socio-économiques des riziculteurs: Les riziculteurs de l'échantillon se répartissent entre environ $14 \%$ de femmes et $86 \%$ d'hommes. Leur âge varie de 20 à 73 ans avec une moyenne se situant à 45 ans. La répartition des riziculteurs par groupe ethnique dans le tableau 1 montre et enfin par niveau de catégories sociales pour d'une part mieux prendre en compte les disparités régionales et pour d'autre part mieux faire ressortir les stratégies de production mises en œuvre par les différents acteurs. Les analyses ont été réalisées avec la version 11 du logiciel STATA.

que les sénoufos constituent plus de la moitié de l'effectif, soit $53,77 \%$; ensuite viennent les riziculteurs baoulé $(16 \%)$, les producteurs malinké $(13 \%)$; les riziculteurs bété représentent environ $6 \%$ du total et les allogènes constituent $12 \%$ de l'effectif de notre échantillon.

Tableau 1 : Répartition des riziculteurs en fonction des ethnies

Table 1: Rice farmers distribution by ethnic group

\begin{tabular}{|l|c|c|c|}
\hline \multicolumn{1}{|c|}{ Ethnie } & Fréquence & Pourcentage & Pourcentage Cumulé \\
\hline Bété & 11 & 5.53 & 5.53 \\
\hline Baoulé & 32 & 16.08 & 21.61 \\
\hline Sénoufo & 105 & 53.77 & 75.38 \\
\hline Malinké & 25 & 12.56 & 87.94 \\
\hline Autres ethnies (Allogènes) & 24 & 12.06 & 100.00 \\
\hline Total & 199 & 100.00 & \\
\hline
\end{tabular}

Désagrégée par zone agro-écologique, la répartition des riziculteurs par catégories d'origine sociale apporte des indications intéressantes à notre analyse. En effet, en zone forestière, les allochtones constituent près des trois quarts des riziculteurs, loin devant les autochtones bété qui constituent environ $13 \%$ et les allogènes $14 \%$ environ. En zone de savane, les allochtones ne représentent plus qu'environ $17 \%$ des riziculteurs, loin derrière les autochtones qui représentent près de $82 \%$ des producteurs, mais bien devant les allogènes qui ne représentent plus que $2 \%$ environ.

Analyse de la dotation et de l'intensité d'utilisation des facteurs de production : Les facteurs de production analysés sont essentiellement la terre, le travail familial et salarié, et les intrants chimiques dont principalement l'engrais NPK et l'urée. Au niveau agrégé, les résultats du tableau 2 ci-dessous indiquent que chaque ménage rizicole dispose en moyenne de 0,90 ha. Une analyse détaillée des disponibilités en terre indique que la superficie médiane s'élève à un hectare, toutefois près des trois quarts des ménages de riziculteurs disposent d'une superficie inférieure à 1 ha, et seuls environ $25 \%$ d'entre eux disposent de superficie de plus de 1 hectare. Par ménage rizicole et par campagne, le facteur travail est utilisé en moyenne à hauteur de 70 hommes-jour de travail familial et 150 hommes-jour de travail salarié. Les quantités moyennes d'engrais NPK et Urée utilisées sont respectivement de $89 \mathrm{~kg} / \mathrm{ha}$ et $80 \mathrm{~kg} / \mathrm{ha}$. Ces résultats indiquent que le volume de travail salarié utilisé est deux fois plus important que celui de travail familial, ils indiquent par ailleurs que les quantités d'engrais appliquées sont largement en dessous des doses recommandées par la vulgarisation qui sont de 150 à 200 $\mathrm{kg} / \mathrm{ha}$ de NPK et de $100 \mathrm{~kg} / \mathrm{ha}$ d'urée. Ainsi moins de $20 \%$ des riziculteurs apportent l'engrais NPK et l'Urée à la dose recommandée. 


\section{Doumbia et Depieu. J. Appl. Biosci. 2014. Analyse des caractéristiques structurelles, des performances technico-économiques de la riziculture irriguée en Côte d'Ivoire}

Tableau 2 : Dotation et niveau d'utilisation des facteurs de production par les ménages rizicoles pour l'ensemble de l'échantillon $(n=199)$

Table 2: Production factors allocation and use intensity by rice farmers at aggregate level (whole sample: $n=199$ )

\begin{tabular}{|l|l|c|c|cc|}
\cline { 3 - 6 } \multicolumn{2}{|l|}{ Superficie (ha) } & Moyenne & Erreur Standard & \multicolumn{2}{|c|}{ Intervalle de Confiance (95\%) } \\
\hline \multirow{2}{*}{ Main d'œuvre (hj) } & Familiale & 0,9358894 & 0,0441751 & {$[0,8487753$} & $1,023004]$ \\
\cline { 2 - 6 } & Salariée & 71,59045 & 4,958319 & {$[61,81256$} & $81,36834]$ \\
\hline \multirow{2}{*}{ Engrais (kg/ha) } & NPK & 89,8051 & 9,130566 & {$[132,7994$} & $168,8107]$ \\
\cline { 2 - 6 } & UREE & 79,79688 & 6,799465 & {$[75,64244$} & $102,4598]$ \\
\hline
\end{tabular}

Les résultats de l'analyse par zone agro-écologique du tableau 3 indiquent une dotation plus importante en terre en zone forestière qu'en zone de savane. En effet, chaque ménage rizicole dispose en moyenne d'environ un hectare et trois quarts d'hectare pour la production de paddy respectivement en zone de forêt et en zone de savane. Le volume de travail familial utilisé est deux fois plus important en zone de forêt qu'en zone de savane ; les quantités de travail salariées sont par contre légèrement plus élevées en zone de savane qu'en zone de forêt. Les quantités d'engrais NPK utilisées sont nettement plus élevées en zone de savane qu'en zone de forêt, ces quantités sont respectivement de l'ordre de 156 $\mathrm{kg} / \mathrm{ha}$ et $26 \mathrm{~kg} / \mathrm{ha}$. On observe la même tendance pour la consommation d'urée que pour celle de NPK. Un usage plus important du travail familial et de la terre est fait en zone de forêt, tandis qu'en zone de savane un usage plus intensif est fait du travail salarié et de l'engrais. La disponibilité moyenne en terre est la plus élevée au niveau des allochtones, suivent ensuite les allogènes, les autochtones étant ceux qui disposent en moyenne par ménage des plus petites parcelles cultivées en riz. Pour ce qui est du travail familial, les allogènes disposent du volant de main-d'œuvre le plus important, suivent les allochtones puis les autochtones. Ce sont les allochtones qui font le plus grand usage de la main-d'œuvre salariée, le niveau de recours à ce type de travail étant du même ordre de grandeur pour les autochtones et les allogènes. La consommation moyenne par ménage de l'engrais NPK est la plus importante au niveau des autochtones, le niveau de consommation de ce type d'engrais est sensiblement du même ordre de grandeur pour les allochtones et pour les allogènes. Pour la consommation moyenne d'urée, on observe la même tendance que celle notée au niveau de l'engrais NPK. II est à noter que ce sont les autochtones qui font le plus grand usage de l'engrais, les allochtones ayant le plus grand recours à la terre et au travail salarié, tandis que les allogènes ont surtout recours au travail familial.

Tableau 3 : Dotation en ressources productives et niveau d'utilisation des facteurs de production par les ménages rizicoles par zone agro écologique

Table 3: Production factors allocation and use intensity according to agro ecological zones

\begin{tabular}{|c|c|c|c|c|c|c|}
\hline & Moyenne & Erreur Standard & \multicolumn{2}{|c|}{ Intervalle de Confiance (95\%) } \\
\hline \multirow{2}{*}{\multicolumn{2}{|c|}{ Superficie (ha) }} & Forêt & 1,082718 & 0,0626926 & {$[0,9590875$} & $1,206349]$ \\
\hline & & Savane & 0,7783542 & 0,0582975 & {$[0,6633904$} & $0,8933179]$ \\
\hline \multirow{4}{*}{$\begin{array}{l}\text { Main } \\
\text { d'œuvre } \\
\text { (hj) }\end{array}$} & \multirow[b]{2}{*}{ Familiale } & Forêt & 94,15534 & 8,142973 & {$[78,09725$} & $110,2134]$ \\
\hline & & Savane & 47,38021 & 4,232153 & {$[39,03433$} & $55,72609]$ \\
\hline & \multirow{2}{*}{ Salariée } & Forêt & 132,052 & 11,64636 & {$[109,0852$} & $155,0188]$ \\
\hline & & Savane & 170,9256 & 13,98911 & {$[143,3388$} & $198,5124]$ \\
\hline \multirow{4}{*}{$\begin{array}{l}\text { Engrais } \\
\text { (kg/ha) }\end{array}$} & \multirow{2}{*}{ NPK } & Forêt & 26,12806 & 4,744412 & {$[16,772$} & $35,48413]$ \\
\hline & & Savane & 156,5623 & 9,006723 & {$[138,8009$} & $174,3237]$ \\
\hline & \multirow{2}{*}{ UREE } & Forêt & 41,97026 & 5,0847 & {$[31,94314$} & $51,99738]$ \\
\hline & & Savane & 120,3817 & 9,442155 & {$[101,7616$} & $139,0018]$ \\
\hline
\end{tabular}

Les résultats du tableau 4 indiquent que les allochtones et les allogènes sont mieux dotés en ressources terre que les autochtones. On observe la même tendance de manière plus accentuée au niveau de la dotation en travail familial que celle de la ressource terre. La dotation en travail familial est deux fois plus importante au niveau 


\section{Doumbia et Depieu. J. Appl. Biosci. 2014. Analyse des caractéristiques structurelles, des performances technico-économiques de la riziculture irriguée en Côte d'Ivoire}

des allogènes qu'au niveau des allochtones. Le recours à la main d'œuvre salariée semble plus équilibrée entre les trois catégories d'origine sociale, avec toutefois un recours un peu plus important au travail salarié de la part des allochtones comparativement aux deux autres catégories d'origine sociales que sont les autochtones et les allogènes. Le niveau de consommation de l'engrais NPK est nettement plus important en ce qui concerne les autochtones qu'en ce qui concerne les allochtones et les allogènes. La tendance de consommation de l'urée est assez similaire à celle du NPK au niveau des trois groupes de catégories d'origine sociale.

Tableau 4 : Dotation en ressources productives des ménages rizicoles par catégories d'origine sociale Table 4: Rice farmers' productive resources allocation according to their social origin

\begin{tabular}{|c|c|c|c|c|c|c|}
\hline & \multirow{2}{*}{$\begin{array}{c}\text { Moyenne } \\
0,7337582\end{array}$} & \multirow{2}{*}{$\begin{array}{c}\begin{array}{c}\text { Erreur } \\
\text { Standard }\end{array} \\
0,0595964\end{array}$} & \multicolumn{2}{|c|}{ Intervalle de Confiance (95\%) } \\
\hline \multirow{3}{*}{\multicolumn{2}{|c|}{ Superficie (ha) }} & Autochtones & & & {$[0,6162332$} & $0,8512833]$ \\
\hline & & Allochtones & 1,139891 & 0,0656968 & {$[1,010336$} & $1,269446]$ \\
\hline & & Allogènes & 0,9125 & 0,1309023 & {$[0,6543583$} & $1,170642]$ \\
\hline \multirow{6}{*}{$\begin{array}{l}\text { Main } \\
\text { d'œuvre } \\
\text { (hj) }\end{array}$} & \multirow{3}{*}{ Familiale } & Autochtones & 47,06044 & 4,284444 & {$[38,61144$} & $55,50944]$ \\
\hline & & Allochtones & 90,78261 & 9,118242 & {$[72,80128$} & $108,7639]$ \\
\hline & & Allogènes & 100,75 & 9,1378833 & {$[82,73003$} & $118,77]$ \\
\hline & \multirow{3}{*}{ Salariée } & Autochtones & 139,2397 & 11,61045 & {$[116,3437$} & $162,1358]$ \\
\hline & & Allochtones & 165,4086 & 15,33219 & {$[135,1732$} & $195,6439]$ \\
\hline & & Allogènes & 132,6128 & 27,05373 & {$[79,26238$} & $185,9632]$ \\
\hline \multirow{6}{*}{$\begin{array}{l}\text { Engrais } \\
\text { (kg/ha) }\end{array}$} & \multirow{3}{*}{ NPK } & Autochtones & 138,32 & 10,91924 & {$[116,7871$} & $159,853]$ \\
\hline & & Allochtones & 44,83178 & 6,447269 & {$[32,11765$} & $57,54591]$ \\
\hline & & Allogènes & 63,09524 & 18,88572 & {$[25,85227$} & $100,3382]$ \\
\hline & \multirow{3}{*}{ UREE } & Autochtones & 107,7391 & 10,64231 & {$[86,75232$} & $128,726]$ \\
\hline & & Allochtones & 51,53037 & 5,769593 & {$[40,15263$} & $62,9081]$ \\
\hline & & Allogènes & 83,40774 & 12,77497 & {$[58,21526$} & $108,6002]$ \\
\hline
\end{tabular}

Analyse de la productivité agricole en riziculture irriguée : Les résultats de l'étude indiquent qu'au niveau agrégé, la productivité physique de la terre est de 3 tonnes de paddy à l'hectare, tandis que la productivité du travail, exprimée par la valorisation journalière du travail familial est de 1742 F CFA (USD 3.484) par jour, ce qui est sensiblement supérieure au coût d'opportunité de la journée de travail, estimé à 1000 FCFA (USD 2) par jour. (tableau 5). Le rendement moyen de 3 tonnes de paddy à
I'hectare est bas, comparativement au potentiel de rendement des variétés améliorées de la recherche. Toutefois, le rendement présente la particularité intéressante d'être relativement stable au niveau de l'échantillon des producteurs. Le rendement varie dans la fourchette de 2,790 tonnes/ha à 3, 223 tonnes/ha. La variabilité est par contre très importante au niveau de la valorisation journalière du travail. Cette quantité varie de 8900 FCFA/J à +12420 F CFA/J.

Tableau 5 : Niveau de productivité des ménages rizicoles pour l'ensemble de l'échantillon ( $n=199)$

Table 5: Technical and economic performances of rice farmers at aggregate level (whole sample: $n=199$ )

\begin{tabular}{|l|c|c|c|}
\cline { 2 - 5 } \multicolumn{1}{c|}{} & Moyenne & Erreur Standard & \multicolumn{2}{|c|}{ Intervalle de Confiance (95\%) } \\
\hline Rendement (kg/ha) & 3007,111 & 109,976 & {$\left[\begin{array}{ll}2790,236 & 3223986\end{array}\right]$} \\
\hline $\begin{array}{l}\text { Valorisation journalière du } \\
\text { travail familial (FCFA/jour) }\end{array}$ & 1741,763 & 5403,187 & {$\left[\begin{array}{ll}-8939,967 & 12423,49\end{array}\right]$} \\
\hline
\end{tabular}

L'analyse de ces mêmes indicateurs au niveau des zones agro écologiques indiquent qu'en moyenne les rendements sont plus élevés en zone de savane qu'en zone de forêt, la différence de rendement entre les deux zones agro écologiques est d'environ une tonne de paddy à l'hectare, comme il apparaît au tableau 6 . La valorisation de la journée de travail de la main-d'œurre familiale est également beaucoup plus forte en zone de 


\section{Doumbia et Depieu. J. Appl. Biosci. 2014. Analyse des caractéristiques structurelles, des performances technico-économiques de la riziculture irriguée en Côte d'Ivoire}

savane qu'en zone de forêt. Les résultats de l'étude indiquent qu'en moyenne que ce soit le rendement ou la valorisation journalière du travail familial, la productivité est plus élevée en zone de savane qu'en zone de forêt. Ces résultats sont consignés au tableau 7 ; ils indiquent par ailleurs que le rendement de 3 tonnes/ha environ est du même ordre de grandeur au niveau des trois catégories d'origine sociale de producteur. Au niveau de la valorisation journalière du travail familial, on note que ce sont les autochtones qui ont la rémunération la plus élevée, pratiquement le double de celle des allogènes et supérieure d'environ $30 \%$ à celles des allochtones.

Tableau 6 : Niveau de productivité des ménages rizicoles par zone agro écologique

Table 6: Technical and economic performances of rice farmers according to agro ecological zones

\begin{tabular}{|l|l|c|c|cc|}
\cline { 3 - 6 } \multicolumn{2}{c|}{} & Moyenne & Erreur Standard & \multicolumn{2}{|c|}{ Intervalle de Confiance (95\%) } \\
\hline $\begin{array}{l}\text { Rendement } \\
\text { (kg/ha) }\end{array}$ & Forêt & 2576,182 & 121,5955 & {$[2336,393$} & $2815,97]$ \\
\cline { 2 - 6 } & Savane & 3469,462 & 175,7102 & {$[3122,959$} & $3815,966]$ \\
\hline $\begin{array}{l}\text { Valorisation } \\
\text { journalière du } \\
\text { travail familial } \\
\text { (FCFA /jour) }\end{array}$ & Forêt & $-3165,396$ & 10752,42 & {$\left[\begin{array}{ll}-24422,2 & 18091,4]\end{array}\right]$} \\
\cline { 2 - 6 } & Savane & 5032,447 & 5464,377 & {$\left[\begin{array}{lll}-5770,251 & 15835,14\end{array}\right]$} \\
\hline
\end{tabular}

Tableau 7 : Niveau de productivité des ménages rizicoles par catégories d'origine sociale

Table 7: Technical and economic performances of rice farmers according to their social origin

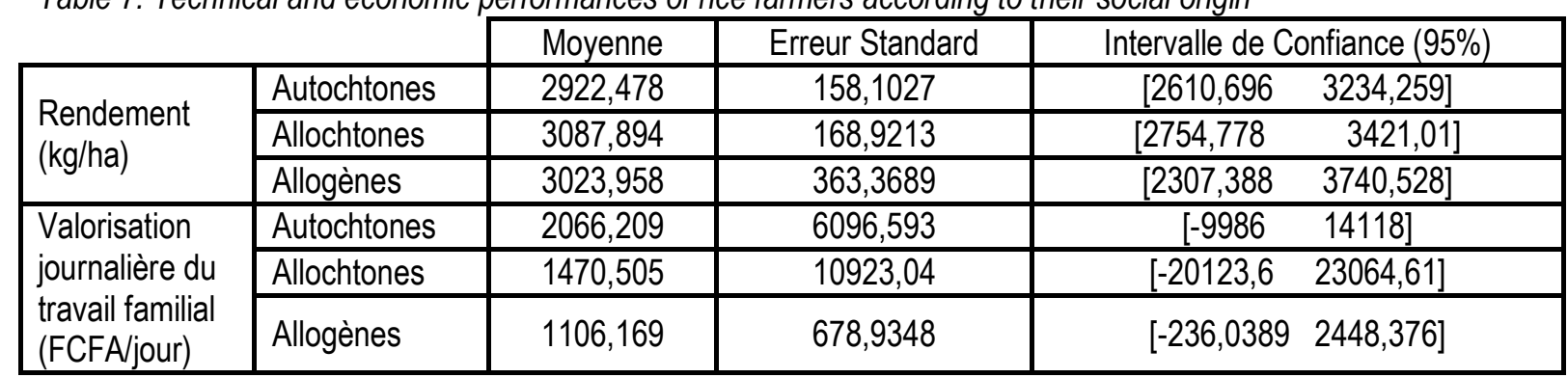

Analyse des résultats économiques dans le cadre de la production de paddy : Les résultats du tableau 8 cidessous indiquent qu'au niveau agrégé la marge brute s'élève en moyenne à 108187,8 FCFA/ha (USD 216.375) tandis que le bénéfice net moyen est de 38475,5 FCFA/ha (USD 76.961) Environ 52,74\% des riziculteurs ont un bénéfice net par hectare positif. La différence, soit $47,6 \%$ des ménages agricoles aboutissent à des résultats de fin de campagne négatifs. Les résultats économiques moyens d'ensemble sont caractérisés par leur faiblesse relative, particulièrement en ce qui concerne le bénéfice net moyen par hectare. L'analyse des résultats économiques menée au niveau des deux zones agro écologiques de production indique que la marge brute par hectare est beaucoup plus élevée en zone de savane qu'en zone de forêt. Le bénéfice net moyen à l'hectare est négatif en zone de forêt, ce bénéfice net moyen positif en zone de savane est de l'ordre de 70000 FCFA (USD 140) par hectare. Par ailleurs, le pourcentage de riziculteurs aboutissant à un bénéfice net par hectare positif en fin de campagne est plus important en zone de savane $(57,3 \%)$ qu'en zone de forêt $(45,61 \%)$ (tableau 9 ). L'ensemble de ces résultats indiquent que la production de paddy est beaucoup plus rentable en zone de savane qu'en zone de forêt. Les résultats du tableau 10 indiquent que la marge brute moyenne à l'hectare est la plus élevée au niveau des allochtones, suivi des allogènes, les autochtones affichent le niveau de marge brute le plus bas. Le bénéfice net moyen à l'hectare est le plus élevé au niveau des allochtones, il est du même ordre de grandeur entre autochtones et allogènes. Les allochtones représentent la catégorie sociale où le pourcentage de riziculteurs ayant un bénéfice net moyen à l'hectare positif est le plus élevé. Ce type de producteur représente un peu plus de $60 \%$ au niveau des allochtones et s'élève respectivement à environ $47 \%$ et $41 \%$ au niveau des autochtones et des allogènes. II résulte de cette analyse que ce sont les allochtones qui obtiennent les meilleurs résultats économiques en riziculture irriguée, comparativement aux membres des deux autres catégories d'origine sociale. 


\section{Doumbia et Depieu. J. Appl. Biosci. 2014. \\ Analyse des caractéristiques structurelles, des performances technico-économiques de la riziculture irriguée en Côte d'Ivoire}

Tableau 8 : Résultats économiques des ménages rizicoles pour l'ensemble de l'échantillon $(n=199)$

Table 8: Global economic performance of rice farmers at aggregate level (whole sample: $n=199$ )

\begin{tabular}{|l|c|c|c|}
\cline { 2 - 4 } \multicolumn{1}{c|}{} & Moyenne & Erreur Standard & Intervalle de Confiance (95\%) \\
\hline Marge brute/ha (FCFA) & 108187,8 & 20233,21 & {$[68197,71148177,9]$} \\
\hline Bénéfice net/ha (FCFA) & 38475,5 & 21656,87 & {$[-4328,4381279,42]$} \\
\hline Bénéfice net/ha>0 & $52,74 \%$ & 4,14604 & {$[44,5460,93]$} \\
\hline
\end{tabular}

Tableau 9 : Résultats économiques des ménages rizicoles par zone agro écologique

Table 9: Global economic performance of rice farmers according to agro ecological zones

\begin{tabular}{|l|l|l|c|c|}
\cline { 3 - 5 } \multicolumn{2}{c|}{} & \multicolumn{1}{c|}{ Moyenne } & Erreur Standard & Intervalle de Confiance (95\%) \\
\hline FCFA Brute/ha en & Forêt & 94524,92 & 27707,77 & {$[39761,64149288]$} \\
\cline { 2 - 5 } & Savane & 116938,2 & 28132,67 & {$[61335,15172541,3]$} \\
\hline \multirow{2}{*}{$\begin{array}{l}\text { Bénéfice net/ha en } \\
\text { FCFA }\end{array}$} & Forêt & $-11132,97$ & 32274,41 & {$[-74922,0452656,1]$} \\
\cline { 2 - 5 } & Savane & 70247,21 & 28528,18 & {$[13862,42126632]$} \\
\hline \multirow{2}{*}{ Bénéfice net/ha>0 } & Forêt & $45,61 \%$ & 0,0665 & {$[32,4558,76]$} \\
\cline { 2 - 5 } & Savane & $57,30 \%$ & 0,05272 & {$[46,8867,72]$} \\
\hline
\end{tabular}

Tableau 10 : Résultats économiques des ménages rizicoles par catégories d'origine sociale

Table 10: Global economic performance of rice farmers according to their social origin

\begin{tabular}{|c|c|c|c|c|}
\hline & & Moyenne & Erreur Standard & Intervalle de Confiance (95\%) \\
\hline \multirow{3}{*}{$\begin{array}{l}\text { Marge } \\
\text { Brute/ha en } \\
\text { FCFA }\end{array}$} & Autochtones & 65131,93 & 26548,17 & {$\left[\begin{array}{ll}12660,54 & 117603,3\end{array}\right]$} \\
\hline & Allochtones & 160941 & 32852,66 & {$[96009,08$} \\
\hline & Allogènes & 125901,3 & 76624,8 & {$[-25544,51$} \\
\hline \multirow{3}{*}{$\begin{array}{l}\text { Bénéfice } \\
\text { net/ha en } \\
\text { FCFA }\end{array}$} & Autochtones & 19204,3 & 27008,11 & {$[-34176,14$} \\
\hline & Allochtones & 68018,62 & 38633,82 & {$[-8339,559$} \\
\hline & Allogènes & 17734,63 & 75523,67 & {$[-131534,8$} \\
\hline \multirow{3}{*}{$\begin{array}{l}\text { Bénéfice } \\
\text { net/ha>0 }\end{array}$} & Autochtones & $47,36 \%$ & 0,0576 & {$[0,35590,5876]$} \\
\hline & Allochtones & $62,06 \%$ & 0,0642 & {$[0,49360,7477]$} \\
\hline & Allogènes & $41,66 \%$ & 0,1486 & {$[0,12280,7104]$} \\
\hline
\end{tabular}

\section{DISCUSSION}

Le fait que les hommes soient plus prépondérants dans la riziculture irriguée comparativement aux femmes est une réalité incontestable en Côte d'lvoire. Dans de nombreuses régions du pays et particulièrement en zone de forêt, la pratique de la riziculture pluviale relève quasi exclusivement des femmes, tandis que les hommes, particulièrement les allochtones et les allogènes s'investissent plutôt dans la riziculture irriguée. Les autochtones masculins s'adonnent de préférence aux cultures d'exportation comme le café et le cacao et plus récemment l'hévéa (Doumbia et al, 2013 ; Depieu et al, 2010). Par ailleurs, la répartition en pourcent des producteurs entre les différentes catégories d'origine sociale est assez conforme à la réalité, car elle épouse assez bien l'histoire du développement agricole en Côte
d'Ivoire, construite en partie à partir de migrations successives depuis l'indépendance du pays orientées essentiellement vers la zone forestière. En effet, comparativement à la zone de savane, la zone forestière concentre le plus de populations allochtones et allogènes nées des flux migratoires à la recherche de terre et d'opportunités économiques plus importantes dans cette partie du pays (Chaléard, JL, 1988 ; Dozon, JP, 1985). En fonction des stratégies de production des différents acteurs, on note une plus grande combinaison des facteurs terre et travail familial en zone de forêt, tandis que la production en ayant davantage recours au travail salarié et aux intrants chimiques paraît plus capitalistique en zone de savane. En ce qui concerne les différents acteurs, on peut noter de manière schématique qu'une 
certaine forme d'intensification est recherchée par une utilisation plus accrue d'engrais par les autochtones, les allochtones et les allogènes développent davantage des stratégies de production moins capitalistique par une plus grande utilisation de la terre par les premiers et du travail familial par les seconds. Dans l'ensemble, la productivité demeure faible en riziculture irriguée particulièrement de par la faiblesse des rendements moyens, même si la valorisation journalière du travail est supérieure en moyenne au coût d'opportunité de la journée de travail familial. En effet, le rendement moyen obtenu par les riziculteurs en écologie irriguée est faible comparé au rendement potentiel des variétés améliorées disponibles au niveau de la recherche. Les résultats d'essais expérimentaux en riziculture irriguée indiquent des résultats potentiels de plus de 8 à 10 tonnes/ha de paddy (CNRA, 2005). Par rapport à ces potentiels de production on peut estimer en moyenne le « yield gap » à environ 6

\section{CONCLUSION}

Une enquête socio-économique, avec pour objectif de mieux comprendre les conditions de la production du paddy en écologie irriguée en Côte d'Ivoire a été menée. Les résultats auxquels nous sommes parvenus indiquent que la production de paddy est le fait de plusieurs acteurs, inégalement dotés en ressources productives et mettant en œuvre des stratégies de production variées. Au-delà des acteurs, le clivage agro écologique s'est avéré un facteur déterminant de la production du paddy. De manière générale, l'étude conclut à la faiblesse des performances technico-économiques en partie à cause du non respect des recommandations de la vulgarisation et des prix d'achat peu rémunérateur du paddy aux producteurs. L'étude détermine au plan technicoéconomique un certain nombre d'indicateurs clés à suivre et qui constituent par ailleurs autant de cibles dans la perspective d'une amélioration des performances de la riziculture irriguée. Le fait que l'estimation de ces indicateurs présente une grande variabilité n'enlève rienà leur intérêt. II s'agit des indicateurs de productivité que sont le rendement et la valorisation journalière du travail

\section{BIBLIOGRAPHIE}

Adesina, A, 1993. Economics of Rice Production in West Africa, WARDA State of the Arts Paper.

Anonyme, 2012. Synthèse de la stratégie révisée de développement de la filière riz 2012-2020. Office National de Développement de la Riziculture (ONDR). Ministère de l'Agriculture/République de Côte d'Ivoire. 13p. tonnes/ha ce qui constitue une bonne indication de la marge de progrès à réaliser par les riziculteurs ivoiriens. De plus, les différentes stratégies de production mises en

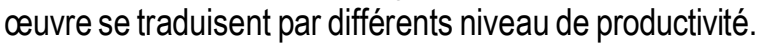
On note en zone de savane des rendements plus élevés et une meilleure valorisation de la journée de travail qu'en zone forestière, tandis que les autochtones démontrent une productivité du travail plus élevée que les allochtones et les allogènes (Anonyme, 2011). Les performances économiques s'avèrent plutôt faibles dans l'ensemble en riziculture irriguée. Seuls un peu plus de la moitié des riziculteurs de notre échantillon peuvent être considérés comme des producteurs efficaces. La zone de savane se caractérise par des performances économiques supérieures à celles obtenues en zone de forêt. Les allochtones s'avèrent dans l'ensemble être des producteurs plus efficaces que les autochtones et les allogènes. (Kore, $A, 2005)$

familial, des résultats économiques d'ensemble, marge brute et bénéfice net par hectare. Par ailleurs, l'ensemble des résultats auxquels nous avons abouti permet de formuler les recommandations suivantes: (1) étant donné la faiblesse des rendements, et donc de l'importance de l'écart entre les rendements moyens obtenus par les producteurs et les rendements potentiels des nouvelles variétés de riz, la priorité devra être donnée dans un premier temps à l'amélioration des rendements plutôt qu'à une augmentation des superficies rizicultivées à travers des aménagements hydro agricoles coûteux. Dans cette démarche, une place de choix devra être réservée à l'appui aux producteurs par le renforcement de la recherche et de la vulgarisation et le transfert de technologies; (2) en matière de politique agricole, la suppression des subventions à la production dans les conditions actuelles de rapport des prix, s'avère contre productive. Des facteurs de production essentiels tels que les semences et les engrais devraient bénéficier d'un minimum de soutien.

Anonyme, 2011. Analyse de la compétitivité du riz et du maïs en Côte d'Ivoire. Rapport d'étude, Abidjan, Côte d'Ivoire, septembre $2011 ; 74 p$.

Chaléard, JL. 1988. La place des cultures vivrières dans les systèmes de production en agriculture de plantation : le cas du département d'Agboville (Côte d'Ivoire) ; Cahiers Sciences Humaines. 24(1) $1988: 35-49$ 
Chohin-Kuper, A ; Kelly, V; Mariko, D. 2000. 20 ans de réforme économiques en Afrique subsaharienne. Comment les producteurs de la zone de l'Office du Niger ont-ils réagi ? Actes du symposium "Small farming systems, markets and competitiveness.

CNRA. 2005. Fiche technique." Bien cultiver le riz irrigué en Côte d'Ivoire". $4 p$

Depieu ME, Doumbia S, Keli ZJ et Zouzou M, 2010. Typologie des exploitations en riziculture de la région de Saïoua, en zone forestière de la Côte d'Ivoire. Journal of Applied Biosciences 35 :2301-2309.

Doumbia, S et Depieu, M.E. 2013. Perception paysanne du changement climatique et stratégies d'adaptation en riziculture pluviale. Journal of Applied Biosciences 64 : 4822-4831.

Dozon, JP. 1985. La société Bété. Histoires d'une "ethnie" de Côte d'Ivoire. 370p. Editions KARTHALA, 22-24, boulevard Arago ; 75013. ISBN : 2-86537-121-2 ISSN : 0290-6600

Kore, A. 2005. Intensification Agricole et Répartition des revenus sur les périmètres rizicoles en période de libéralisation du marché du riz au Niger. 27p + Annexes ; projet de recherche sur l'économie des systèmes rizicoles au Niger.

Pearson S.R., J.D. Stryker, C.P., Humphreys (eds), 1981. Rice in West Africa. Policy and Economics. Stanford University Press. 